\title{
Tlx acts as a proangiogenic switch by regulating extracellular assembly of fibronectin matrices in retinal astrocytes
}

\begin{abstract}
Akiyoshi Uemura, ${ }^{1}$ Sentaro Kusuhara, ${ }^{1}$ Stanley J. Wiegand, ${ }^{2}$ Ruth T. Yu, ${ }^{3}$ and Shin-Ichi Nishikawa ${ }^{1}$
${ }^{1}$ Laboratory for Stem Cell Biology, Center for Developmental Biology, Institute of Physical and Chemical Research (RIKEN), Kobe, Japan. ${ }^{2}$ Regeneron Pharmaceuticals Inc., Tarrytown, New York, USA. ${ }^{3}$ Gene Expression Laboratory, The Salk Institute for Biological Studies, La Jolla, California, USA.

In response to hypoxia, hypoxia-inducible factors act as the primary proangiogenic triggers by regulating transcription levels of target genes, including VEGF. However, little is known about the specific factors that control other components of the angiogenic process, particularly formation of matrix scaffolds that promote adhesion and migration of endothelial cells. We show that in the postnatal mouse retina, the orphan nuclear receptor tailless $(T l x)$ is strongly expressed in the proangiogenic astrocytes, which secrete VEGF and fibronectin. $T l x$ expression by retinal astrocytes is controlled by oxygen concentration and rapidly downregulated upon contact with blood vessels. In mice null for $T l x$, retinal astrocytes maintain VEGF expression; however, the extracellular assembly of fibronectin matrices by astrocytes is severely impaired, leading to defective scaffold formation and a complete failure of normal retinal vascular development. This work identifies Tlx as an essential component of the molecular network involved in the hypoxia-inducible proangiogenic switch in retinal astrocytes.
\end{abstract}

\section{Introduction}

During tissue neogenesis in development, increased metabolic activities result in an imbalance between blood supply and oxygen demands in the microenvironment, leading to physiological hypoxia and subsequent activation of specific cell populations in each tissue or organ to induce formation of functional blood vessels. Once the blood supply sufficiently meets the oxygen demands, proangiogenic activities are turned off and blood vessels remain quiescent unless angiogenesis resumes under pathological conditions such as cancer or diabetic retinopathy (1-3). Thus, the extent of angiogenesis is regulated by the switching on or off of proangiogenic activities in response to the microenvironmental oxygen concentration. While the molecular mechanisms controlling the angiogenic processes are vastly heterogeneous, depending on the surrounding cell types (4), hypoxia-inducible factors (HIFs) act as the primary molecular switches to the proangiogenic state in many instances $(5,6)$. Under hypoxic conditions, activated HIFs transcriptionally upregulate expression levels of a series of proangiogenic genes, including VEGF (7-9). Subsequently, vascular sprouts invade the target tissues, utilizing scaffolds composed of extracellular molecules that promote endothelial adhesion, migration, and survival $(10,11)$. Fibronectin, which interacts with endothelial integrin $\alpha 5 \beta 1$, is an essential proangiogenic matrix component as genetic or pharmacological inhibition of fibronectin-integrin $\alpha 5 \beta 1$ interactions disrupts angiogenesis in both developmental and pathological conditions (12-15). To date, however, the molecular mechanisms that control formation of proangiogenic scaffolds under hypoxic conditions remain poorly understood.

Nonstandard abbreviations used: GFAP, glial fibrillary acidic protein; HIF, hypoxiainducible factor; ISH, in situ hybridization; Tlx, tailless.

Conflict of interest: S.J. Wiegand is an employee of Regeneron Pharmaceuticals Inc., which has a commercial interest in developing VEGF Trap as a drug.

Citation for this article: J. Clin. Invest. 116:369-377 (2006). doi:10.1172/JCI25964.
In the mouse retina, it is well established that astrocytes act as the primary proangiogenic cell type required to establish the retinal vascular system. Prior to the postnatal onset of retinal vascular development, retinal neurons induce an influx of astrocytes via signaling molecules such as PDGF-A. Subsequently, retinal astrocytes secrete VEGF and fibronectin, thereby guiding retinal angiogenesis over the astrocyte network (16-22). After completion of blood vessel formation, astrocytes lose their proangiogenic properties but continue to play a role in vascular homeostasis by promoting the formation and maintenance of the blood-retina barrier (23-25). Thus, retinal astrocytes serve as a unique and simple model to investigate the molecular mechanisms controlling various proangiogenic processes, including the formation of matrix scaffolds. Interestingly, besides being an important stimulus that triggers angiogenesis, hypoxia modulates several aspects of astrocyte behavior, such as mitotic activity (22). However, with the exception of possible HIF involvement, little is known concerning the cell-intrinsic molecular mechanisms regulating astrocyte behavior in response to changes in tissue oxygen concentration.

The orphan nuclear receptor mouse homolog of Drosophila tailless (Tlx, also known as $\mathrm{Nr} 2 \mathrm{e} 1$ ) is a transcription factor that regulates several genes expressed in astrocytes, such as glial fibrillary acidic protein (GFAP) $(26,27)$. In the adult brain, Tlx is expressed in neural stem cells and directly represses the transcription of GFAP, resulting in maintenance of pluripotency by preventing commitment into astrocyte lineages (27). In the developing retina, several types of cells, including retinal progenitor cells, astrocytes, and Müller glia, express Tlx in a spatially and temporally restricted manner, and retinas of Tlx mutant mice display disorganized astrocyte networks and defective blood vessel formation $(26,28,29)$. Taken collectively, the available data indicate that Tlx may play important roles in controlling astrocyte development, although little is known regarding the mechanisms that regulate Tlx expression or the specific role of Tlx in regulating astrocyte function. 
A

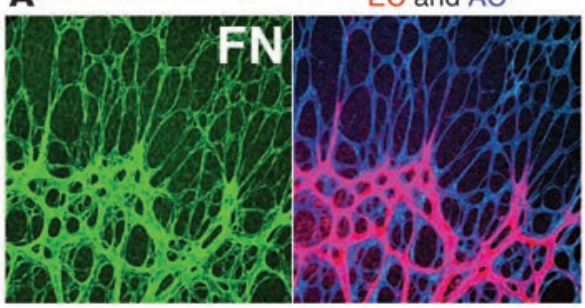

EC
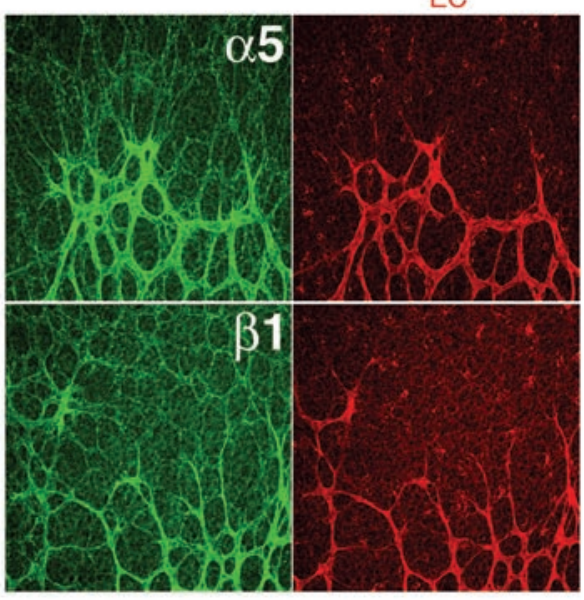

$\mathbf{F}$

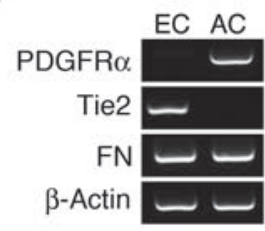

G

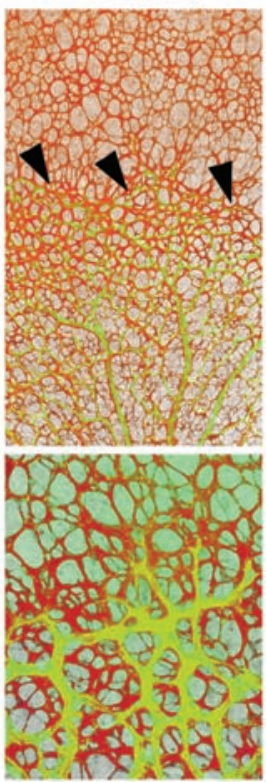

B

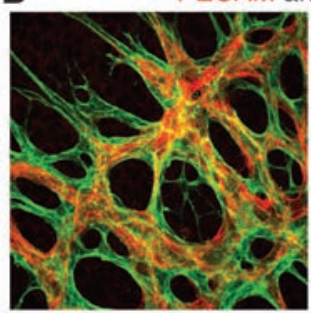

C Control

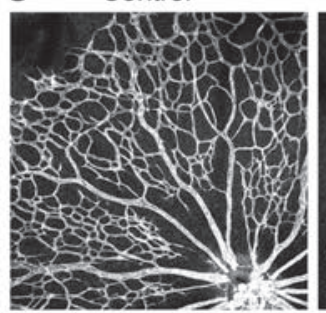

D FN and PDGFR $\alpha$

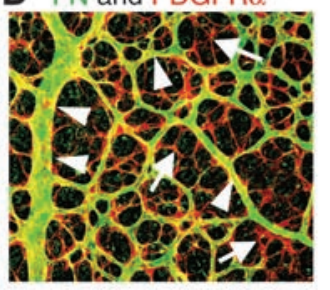

FN
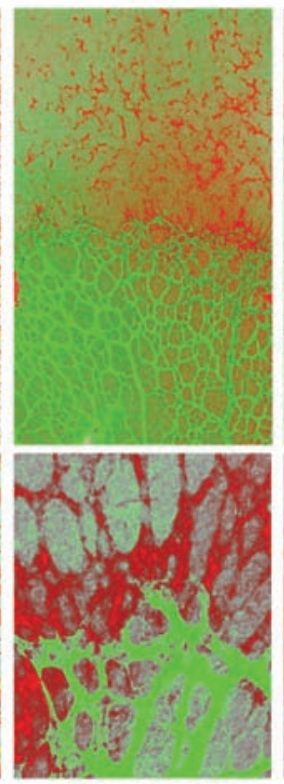

Green: coll IV

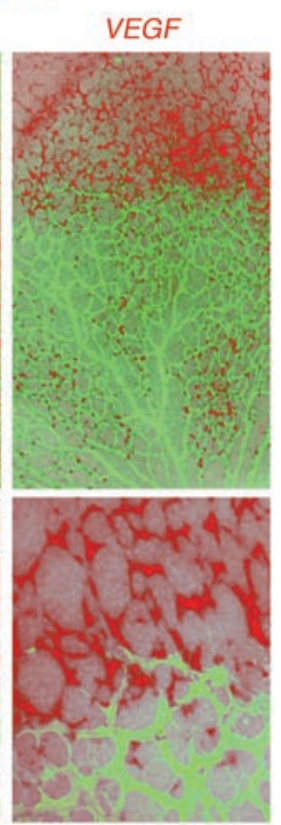

Figure 1

Formation of fibronectin-matrix scaffolds in proangiogenic astrocytes. (A) Triple immunostaining (top) for fibronectin (FN, green), PECAM-1 (red), and PDGFR $\alpha$ (blue) and double immunostaining (middle and bottom) for lectin (red) and integrin $\alpha 5$ or $\beta 1$ (green) at the sprouting vascular edges of P5 retina. (B and C) Retinal vessels 24 (B) or 48 (C) hours after intraocular injections of control IgG (left) or anti-integrin $\beta 1$ antibody (right) at P2. (B) Immunostaining for PECAM-1 (red) and PDGFR $\alpha$ (green) at the sprouting vascular edges. Arrows indicate endothelial cells dissociating from astrocyte (AC) scaffolds. (C) PECAM-1 staining. (D) Immunostaining for PDGFR $\alpha$ (red) and fibronectin (green) in P7 retina. Fibronectin is expressed around blood vessels (arrowheads) but almost absent in astrocyte networks, which are not associated with blood vessels (arrows). (E) Flow cytometric analysis of retinal cells from Tie2-GFP transgenic mice at P7. Endothelial cells and astrocytes were sorted as GFP+ $(0.26 \%)$ and PDGFR $\alpha^{+}(0.43 \%)$ fractions, respectively. (F) RT-PCR from total RNA of GFP+ and PDGFR $\alpha^{+}$ cells sorted by FACS. Fibronectin mRNA is expressed in both endothelial cells and astrocytes. (G) Red immunostaining for PDGFR $\alpha$ (left), ISH for fibronectin (middle), or VEGF (right) in P4 retinas. Arrowheads indicate the growing edge of the retinal vasculature depicted by immunostaining for type IV collagen (coll IV; green). Lower panels are the magnified views of growing vascular edges. Magnification, $\times 200$ (A and D), $\times 400$ (B), $\times 70$ (C), $\times 63(\mathbf{G}$, upper), $\times 252$ (G, lower).
In this study, we show that $T l x$ is specifically expressed by proangiogenic retinal astrocytes and is rapidly downregulated upon contact with blood vessels. The dramatic regulation of Tlx expression is controlled by oxygen concentration. Our analyses of $T l x$ mutant mice reveal that Tlx plays an indispensable role in the formation of the proangiogenic extracellular scaffold, which forms the template for retinal vascular growth through the regulation of the expression and extracellular deposition of fibronectin. Thus, Tlx represents a novel member of the hypoxia-inducible molecular network required for the expression of proangiogenic properties by retinal astrocytes.

\section{Results}

Formation of fibronectin matrix scaffolds in proangiogenic astrocytes. It has been suggested that extracellular matrices deposited by astrocytes forms a proangiogenic scaffold, which plays an important role in retinal vascular development (21). However, the mechanisms that regulate the formation of this scaffold and its func- 
tional significance have not yet been fully defined. In the present study, we first examined the deposition of fibronectin and the expression of its receptor, $\alpha 5 \beta 1$ integrin, at the growing edge of the retinal vasculature. While fibronectin matrices were deposited around the PDGFR $\alpha$-expressing astrocyte networks ahead of sprouting endothelial cells, both $\alpha 5$ and $\beta 1$ integrin subunits were strongly expressed in the endothelial cells themselves (Figure 1A). Such complementary expression patterns suggested that the integrin played a critical role in promoting the adhesion of migrating endothelial cells to the fibronectin scaffolds. We therefore injected P2 neonates intraocularly with a monoclonal antibody against integrin $\beta 1$ subunit to inhibit its binding to extracellular matrices, including fibronectin (30). Twenty-four hours after the intraocular injections, advancement of vascular networks was arrested, and the extension of the endothelial sprouts at the leading edge of the retinal vasculature was decreased (Figure 1B). Furthermore, endothelial cells were no longer strictly associated with the underlying astrocyte network (Figure 1B), leading to enlargement and apparent coalescence of retinal capillaries 48 hours after the treatment (Figure 1C). Thus, integrin-mediated adhesion to the matrix scaffold is an essential part of the development and morphogenesis of the retinal vascular system.

In the course of normal retinal development, fibronectin continued to be present around blood vessels though it was no longer expressed in astrocytes (Figure 1D). This indicates that production of fibronectin associated with blood vessels may shift from astrocytes to endothelial and/or mural cells in the processes of retinal vascular development. Indeed, endothelial cells isolated from P7 retinas also expressed the fibronectin mRNA (Figure 1, $\mathrm{E}$ and $\mathrm{F}$ ). To precisely define the expression pattern of fibronectin in retinal astrocytes, we performed in situ hybridization (ISH) on whole-mount retinas in conjunction with immunolabeling for PDGFR $\alpha$ or type IV collagen to depict astrocyte or vascular networks, respectively. While astrocytes that form a simple plexus in the unvascularized areas of the retina strongly expressed fibronectin, its expression became dramatically downregulated upon contact with blood vessels (Figure $1 \mathrm{G}$ ). Interestingly, this pattern of fibronectin expression was very similar to that of $\operatorname{VEGF}(19,22,31$, 32 ), revealing that in the proangiogenic state, retinal astrocytes synchronously express these 2 genes.

Tlx is specifically expressed in proangiogenic astrocytes. While retinal astrocytes strongly express VEGF and fibronectin in the unvascularized areas, it is reported that GFAP is expressed only at very low levels in these astrocytes but strongly expressed in astrocytes associated with blood vessels $(22,31)$. This indicates that the expression of GFAP is inversely correlated with that of proangiogenic mol-

\section{Figure 2}

TIX expression in proangiogenic astrocytes. (A) Dual immunostaining for GFP and GFAP in retinas of GFAP-GFP transgenic mice at PO and P2. While the 2.2-kb GFAP promoter is uniformly activated in all astrocytes, GFAP expression is seen only in astrocytes around the optic discs. (B) Whole-mount ISH for TIx in PO and P2 retinas. Uniform TIx expression in retinal astrocytes at $\mathrm{PO}$ sequentially becomes downregulated in a central-to-peripheral manner. (C) ISH for TIx or GFAP (red) in conjunction with immunostaining for type IV collagen (green) or PDGFR $\alpha$ (yellow) on P4 retina. Lower panels are the magnified views of growing vascular edges. TIx mRNA is specifically expressed in proangiogenic astrocytes and displays a pattern complementary to that of GFAP. Magnification, $\times 63$ (A, B, and upper left and upper right panels of $\mathbf{C}$ ), $\times 252$ (lower and middle panels of $\mathbf{C}$ ). ecules, suggesting that the molecular mechanisms responsible for regulating GFAP expression might also be involved in regulating the expression of proangiogenic molecules in retinal astrocytes. Therefore, we further examined the regulation of GFAP expression in retinal astrocytes using transgenic mice in which GFP expression was driven by a $2.2-\mathrm{kb} 5^{\prime}$ promoter region of the human GFAP gene (33). The 2.2-kb GFAP promoter was active in all astrocyte populations in the early postnatal period, as shown by staining for GFP (Figure 2A). In contrast, expression of GFAP protein was detectable only in astrocytes near the optic disc (Figure 2A), consistent with the expression pattern of GFAP mRNA in retinal whole mounts (data not shown). This discrepancy suggests that GFAP expression is negatively regulated in retinal astrocytes during the early postnatal stages. A good candidate molecule in this regard is the orphan nuclear receptor Tlx, which has been shown to repress GFAP expression in neural stem cells (27). In the developing retina, we have reported that Tlx is transiently expressed in astrocytes during late embryonic and early postnatal stages (29). To determine the precise expression pattern of the Tlx gene and its relation
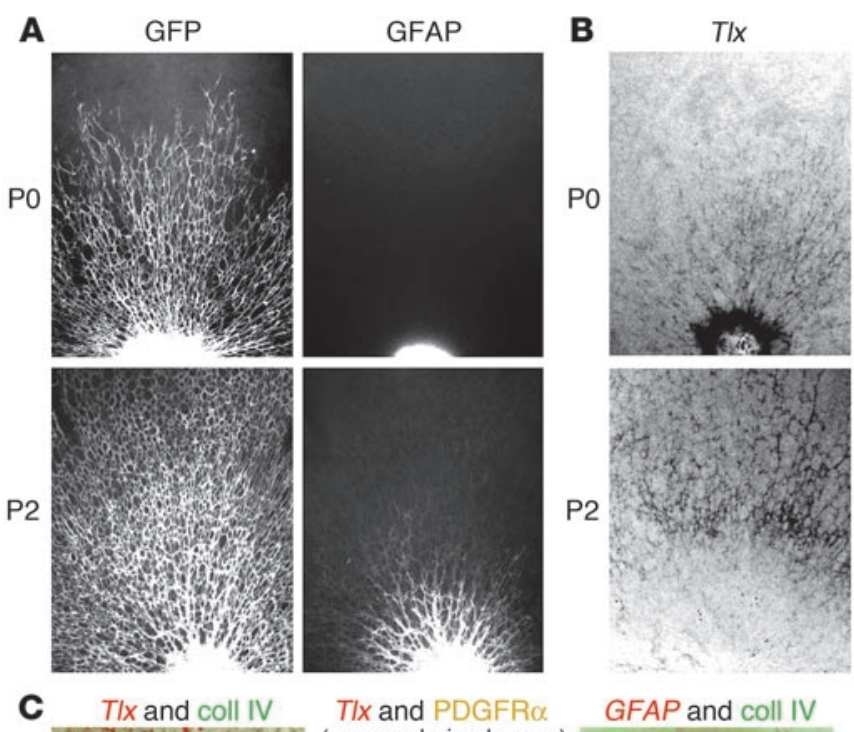

TIx and PDGFR $\alpha$
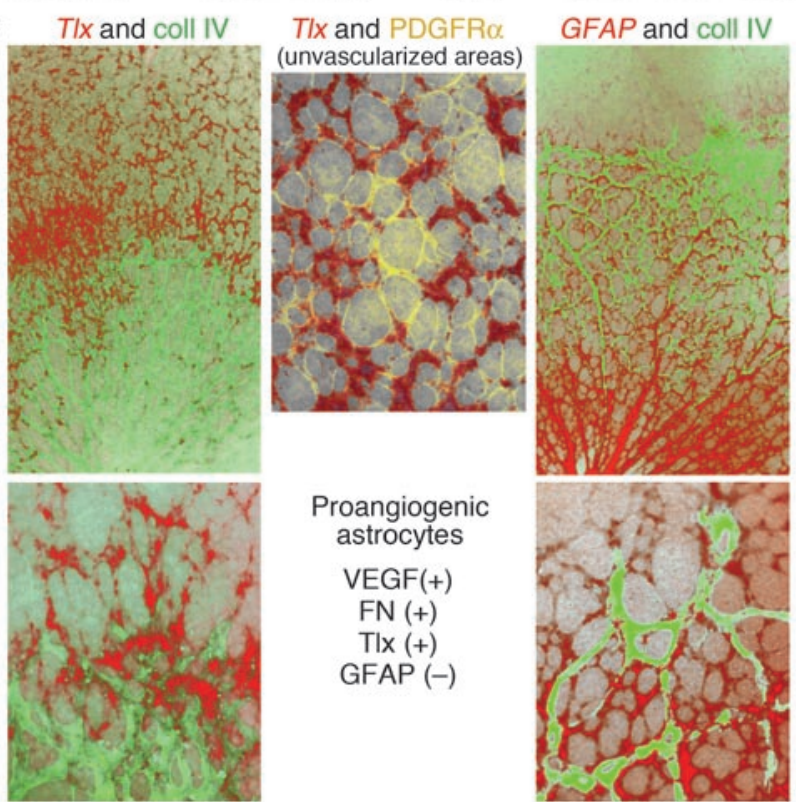
A

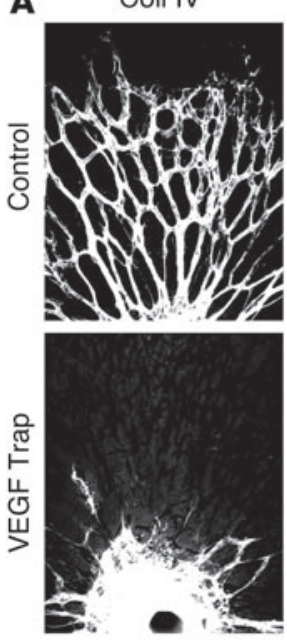

B

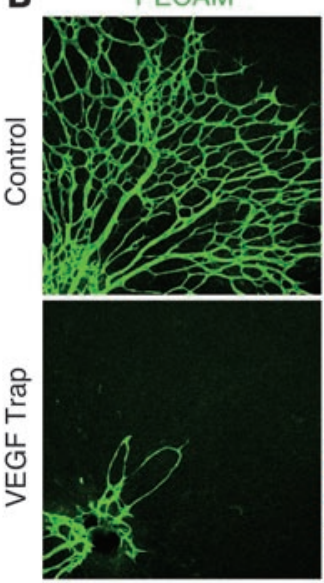

$T / x$

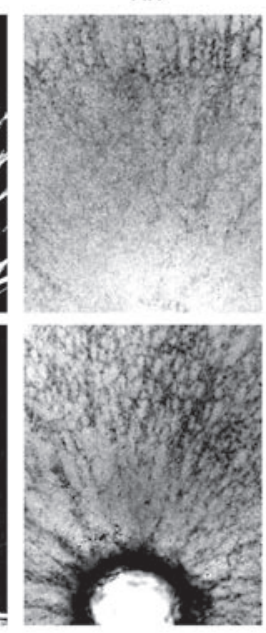

GFAP

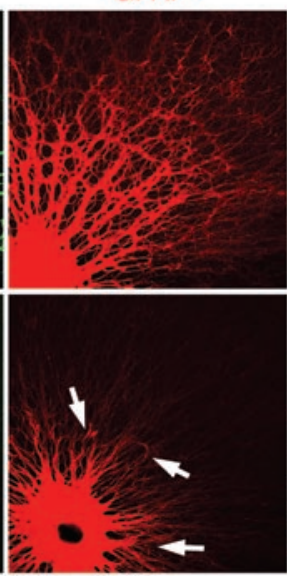

VEGF
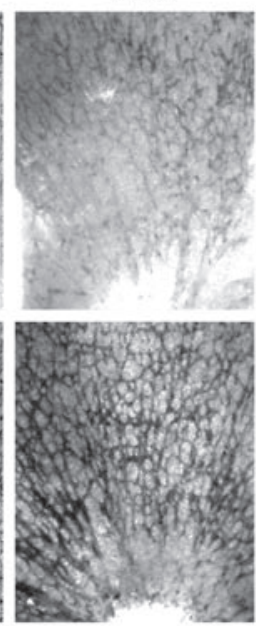

PDGFRo.

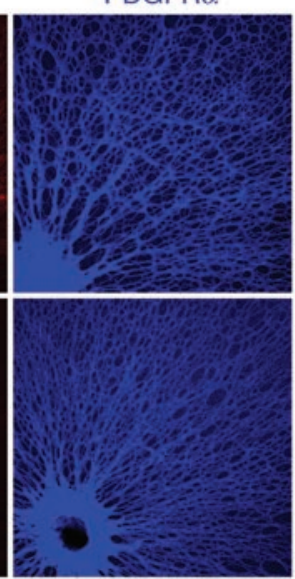

FN
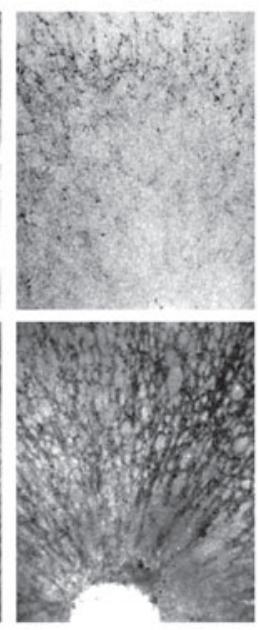

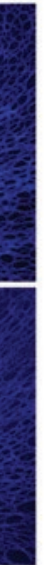

to GFAP in retinal astrocytes, we performed whole-mount ISH in mouse retinas from different developmental stages (Figure 2B). At P0, when the endogenous GFAP expression was barely detectable, Tlx was almost uniformly expressed in the retinal astrocytes. However, during the course of postnatal development, Tlx expression in astrocytes became downregulated in the central retina. To determine whether the transition of Tlx expression in retinal astrocytes was closely correlated with the process of blood vessel formation, we evaluated the pattern of Tlx expression in conjunction with staining for type IV collagen or PDGFR $\alpha$ (Figure 2C). We found that while Tlx was strongly expressed in astrocytes in the unvascularized areas of the retina, its expression was rapidly downregulated upon contact with blood vessels. This pattern of Tlx expression in astrocytes was in clear contrast to that of GFAP (Figure 2C) and almost identical to those of VEGF and fibronectin (Figure $1 G$ ). Thus, we identified Tlx as one of the genes specifically expressed in proangiogenic astrocytes.

Thx expression is controlled by oxygen concentration in retinal astrocytes. In the developing retina, proangiogenic astrocytes are characterized by the expression of high levels of VEGF, fibronectin, and Tlx and very low levels of GFAP. The rapid downregulation of VEGF, fibronectin, and Tlx and simultaneous upregulation of GFAP expression seem to be inducible rather than programmed events triggered by the association with blood vessels. Indeed, when the initiation

\section{Figure 3}

Blood vessels affect expression levels of proangiogenic genes and GFAP in retinal astrocytes. $P 3$ retinas 48 hours after the intraocular injections of Fc control protein (upper panels) or VEGF Trap (lower panels). (A) Immunostaining for type IV collagen and ISH for TIx, VEGF, or fibronectin. In the absence of blood vessels, genes specific to the proangiogenic state are continually expressed in astrocytes. (B) Immunostaining for PECAM-1 (green), GFAP (red), and PDGFR $\alpha$ (blue). In the retinas treated with VEGF Trap, GFAP is expressed only around the poorly developed blood vessels (arrows). In the absence of blood vessels, astrocyte networks depicted by PDGFR $\alpha$ staining maintain a simple plexus formation characteristic of the proangiogenic state. Magnification, $\times 126(\mathbf{A}), \times 100(B)$.

of retinal vascular growth was delayed by intraocular injections of a VEGF receptor chimeric protein, VEGF Trap (34), astrocytes continued to express $T l x, V E G F$, and fibronectin, indicating that these astrocytes remained in the proangiogenic state (Figure 3A). In contrast, GFAP expression was barely detectable except in a few astrocytes associated with blood vessels restricted to the immediate vicinity of the optic nerve head (Figure 3B). Thus, proangiogenic activities and GFAP expression are likely to be coordinately regulated by microenvironmental changes accompanied by blood vessel formation.

Although the mechanisms regulating Tlx and fibronectin expression in retinal astrocytes remain to be determined, the similarity in their developmental expression patterns to that of VEGF suggested that oxygen concentration might play an important role. To investigate this possibility, we placed $\mathrm{P} 2$ pups in $80 \% \mathrm{O}_{2}$ for 5 hours, then returned them to normal room air $\left(20 \% \mathrm{O}_{2}\right)$. After a 5-hour exposure to hyperoxic conditions, $\mathrm{Tlx}$ mRNA expression in retinal astrocytes was markedly suppressed (Figure 4). On the other hand, 18 hours after the return to room air, Tlx mRNA levels were fully restored. Throughout the course of this experiment, the VEGF expression pattern resembled that of Tlx. Thus, in retinal astrocytes, both Tlx and VEGF appear to be transcriptionally controlled by oxygen concentrations, which would explain their synchronous expression in proangiogenic astrocytes and downregulation upon contact with blood vessels. In contrast, fibronectin mRNA was continuously expressed even under hyperoxic conditions, indicating that fibronectin transcription is not directly regulated by oxygen concentration. Despite the rapid downregulation of Tlx under hyperoxic conditions, GFAP mRNA expression was not remarkably upregulated beyond the limits of the retinal vasculature (Figure 4), even when the period of hyperoxia was extended to 24 hours (data not shown), suggesting that relief of Tlx repression alone is not sufficient for upregulation of GFAP expression.

Complete absence of retinal angiogenesis in $T l x^{-/-}$mice. To investigate the functional roles of Tlx in retinal astrocytes, especially those in the proangiogenic state, we analyzed the histological phenotypes of the retinal astrocytes and blood vessels of $T l x \mathrm{KO}\left(T l x^{-/-}\right)$mice. 


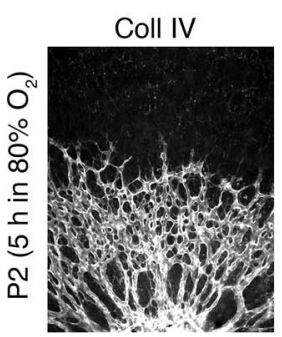

Coll IV

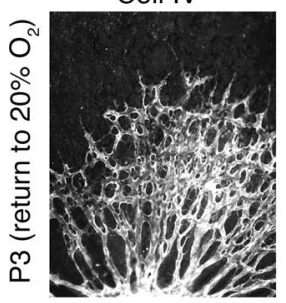

$T / x$

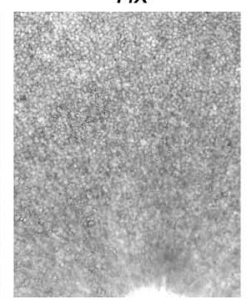

$T / x$

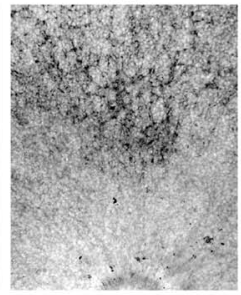

VEGF

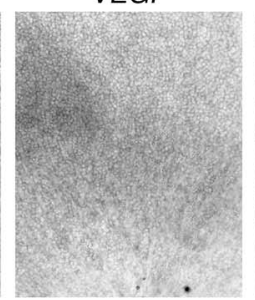

VEGF

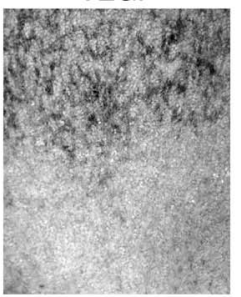

FN

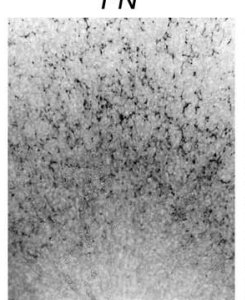

FN

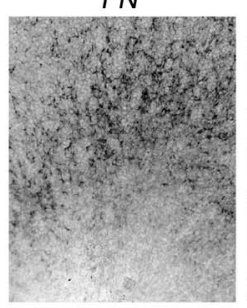

GFAP

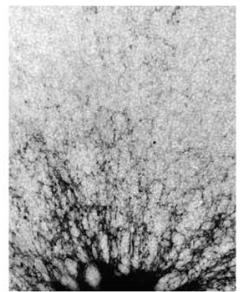

GFAP

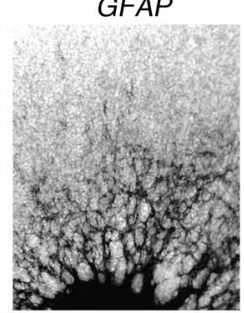

\section{Figure 4}

Oxygen concentration synchronously controls expression of $T / x$ and VEGF but not fibronectin in retinal astrocytes. Immunostaining for type IV collagen and ISH for TIX, VEGF, fibronectin, and GFAP. Expression levels of $T / x$ and VEGF mRNA are dramatically downregulated under hyperoxic conditions (upper panels) whereas they are synchronously restored after the return to room air (lower panels). In contrast, fibronectin expression is continuously maintained even under hyperoxia. GFAP expression is not upregulated beyond the vascularized areas. Magnification, $\times 126$.
The initial formation of the simple astrocyte plexus and subsequent remodeling of the astrocyte network in retinas of Tlx heterozygous $\left(T l x^{+/}\right)$mice was indistinguishable from that of wildtype mice. In contrast, in $T l x^{-/-}$mice, the radial extension of retinal astrocytes was asymmetric and markedly delayed. Moreover, radial astrocyte networks lacked lateral branches and interconnection in $T l x^{-/-}$mice, displaying a spoke-like appearance (Figure 5A). As Tlx has been implicated in cell cycle control $(27,29,35,36)$, we performed BrdU incorporation assays to determine whether the impaired mitotic activity in Tl $x^{-/-}$astrocytes might account for the impaired development of the retinal astrocyte networks (Figure $5 \mathrm{~B})$. Mitotic activity in retinal astrocytes was most pronounced at the leading edge of the developing network. In P5 retinas, the numbers of BrdU-positive astrocytes in this region were decreased to $73.4 \% \pm 19.4 \%$ (mean $\pm \mathrm{SD}, P=0.014$ ) in $T l x^{-/-}$mice compared with $T l x^{+/-}$mice. Because astrocyte proliferation was only modestly decreased, impairments in motility and/or adhesion of $T l x^{-/-}$astrocytes are likely the predominant factors underlying the observed malformation of the astrocyte network in $T l x^{-/-}$retina.

Although an astrocyte network did develop in the retinas of $\mathrm{TlX}^{-}$ mice, albeit abnormally, blood vessels were completely absent (Figure 5C). This was attributable to a failure in normal initiation of retinal angiogenesis, as no vessels grew beyond the optic disc (Figure 5D). Thus, $T l x^{-/}$astrocytes also lacked 1 or more essential proangiogenic properties. Interestingly, retinal astrocytes uniformly expressed GFAP in $T l x^{-/-}$mice, despite the absence of blood vessels

\section{Figure 5}

Morphogenetic defects in network formation and abortive proangiogenic activities in retinal astrocytes of TIX KO mice. (A) Immunostaining for PDGFR $\alpha$ in TIx mutant mice. In $T / X^{-1-}$ mice, astrocyte networks are poorly extended and morphogenetically disorganized, with fewer branchings. (B) Double labeling for BrdU (red) and PDGFR $\alpha$ (green) of migrating astrocytes in $\mathrm{P} 5$ retina. (C and D) Double immunostaining for GFAP (red) and PECAM-1 (green) in $P 5$ retina. GFAP is strongly expressed in all retinal astrocytes of $T / X^{-/}$mice despite the complete absence of blood vessels (C). In $T \mathrm{X}^{-1-}$ mice, blood vessels never sprout from the stem of hyaloid vessels at optic nerve head (arrowheads in D). Nuclear staining by TO-PRO-3 iodide appears in blue. Magnification, $\times 70(\mathbf{A}), \times 400(\mathbf{B})$, $\times 100$ (C), $\times 280$ (D).
A
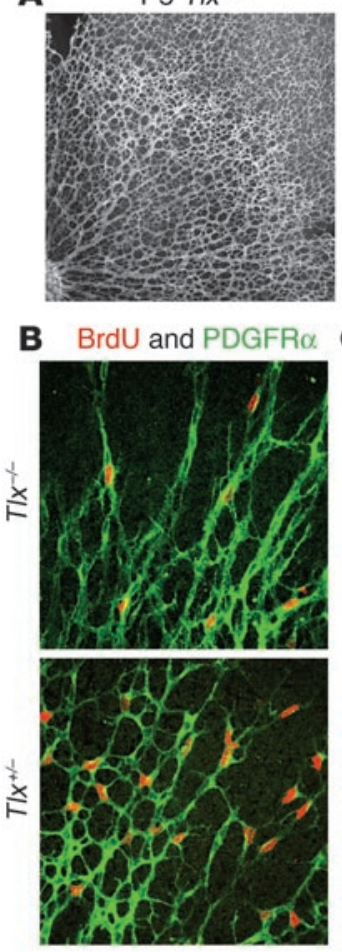

P2 T/X

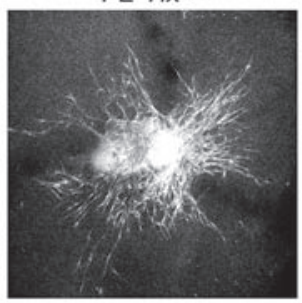

GFAP
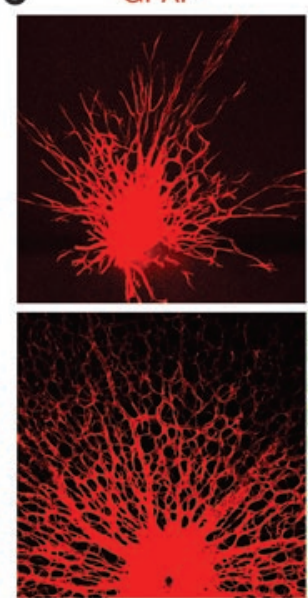

P5 TIX

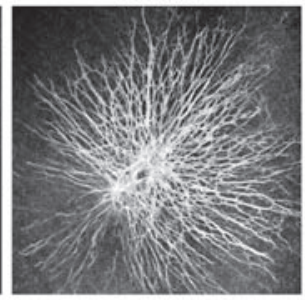

PECAM
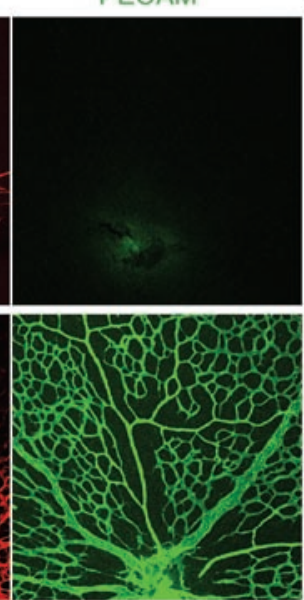

P19 Tlx ${ }^{--}$

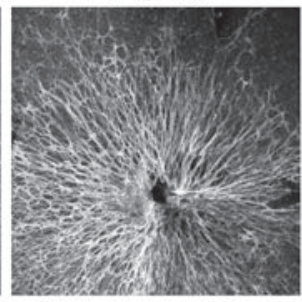

D GFAP and PECAM
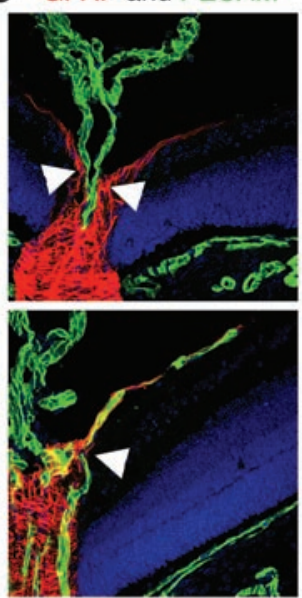

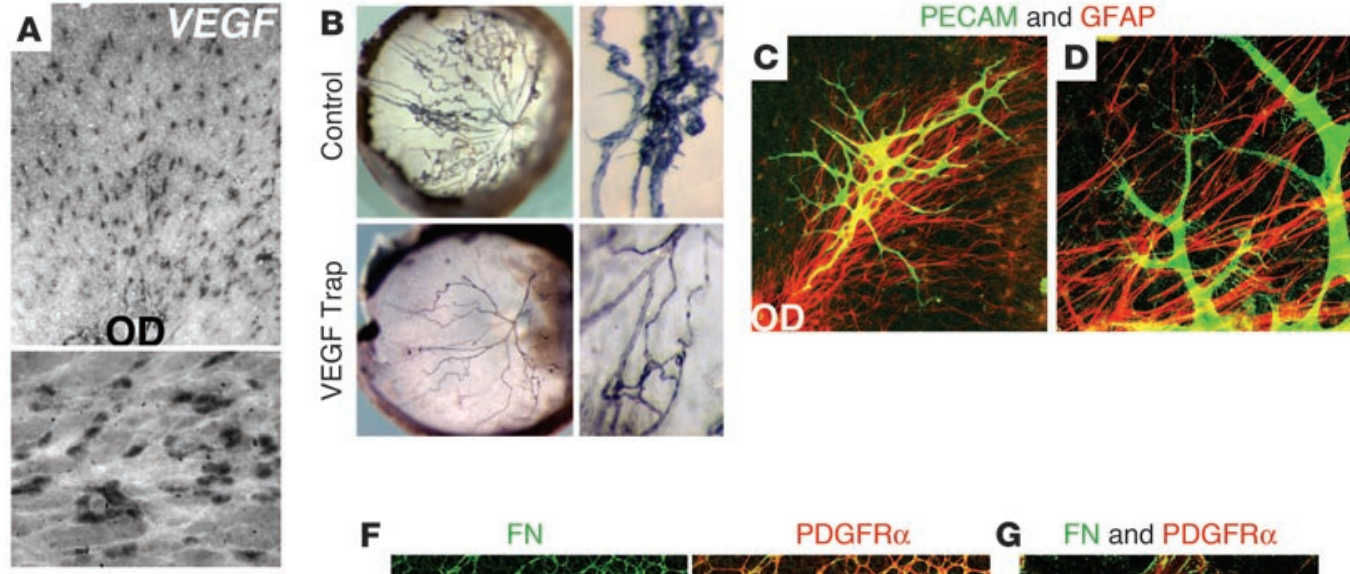

$\mathbf{F}$
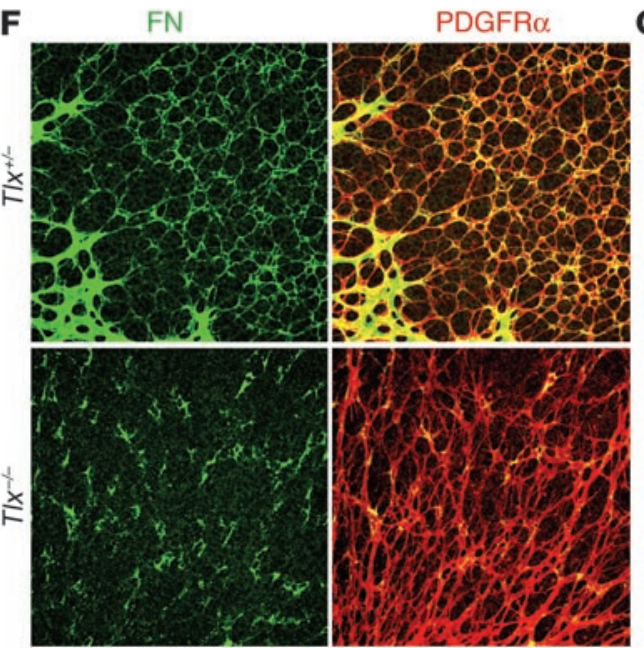

G
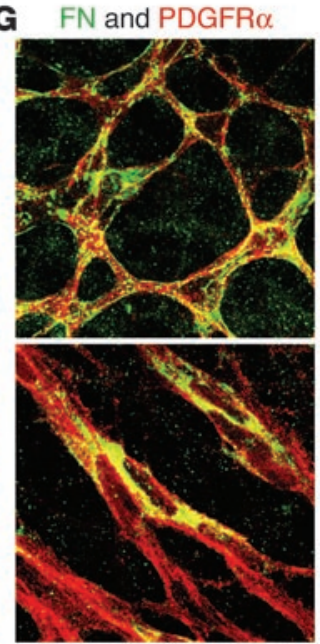

\section{Figure 6}

TIx regulates formation of proangiogenic scaffolds by controlling extracellular deposition of fibronectin matrices in retinal astrocytes. (A and E) Whole-mount ISH for VEGF (A) or fibronectin (E) in P7 retinas of $T / x^{-1-}$ mice. The lower panels are magnifications of ISH (black) with immunostaining for PDGFR $\alpha$ (white). In contrast to the retained VEGF expression over the astrocyte network, fibronectin is expressed only in a small subset of

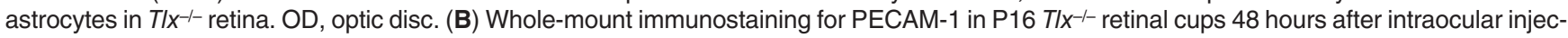
tions of control Fc (upper panels) or VEGF Trap (lower panels). Right panels are the higher magnifications of hyaloid vessels shown in left panels. Persistent hyaloid vessels and their sprouts in T/ $x^{-/-}$eyes regress by neutralization of VEGF signals. (C and D) Immunostaining for PECAM-1 (green) and GFAP (red) in P14 retina of $T / x^{-1-}$ mouse. Ectopic vessels derived from hyaloids target retinal astrocytes (C) but are not guided by the pre-existing astrocyte networks (D). (F and G) Immunostaining for fibronectin (green) and PDGFR $\alpha$ (red) in $T / x^{+/-}$(upper panels) and TIX $x^{-/-}$(lower panels) P7 retinas. In the astrocyte networks of $T / x^{-1-}$ retinas, fibronectin proteins are detected only in a punctate manner $(\mathbf{F})$. Note the retention of fibronectin proteins in perinuclear cytoplasmic regions of $T / x^{-1-}$ astrocytes $(\mathbf{G})$. Magnification, $\times 126$ (upper panels of $\mathbf{A}$ and $\mathbf{E}$ ), $\times 400$ (lower panels of $\mathbf{A}$ and $\mathbf{E}), \times 32$ (B, left panels), $\times 100$ (B, right panels), $\times 150$ (C), $\times 630$ (D), $\times 200(\mathbf{F}), \times 1260$ (G).

(Figure 5C), possibly resulting in part from the loss of Tlx repression on GFAP transcription.

Thx is required for the formation of proangiogenic scaffold through the control of extracellular assembly of fibronectin matrices in retinal astrocytes. As the major known requirement for proangiogenic properties of retinal astrocytes consists of VEGF secretion and extracellular matrix scaffold formation, we investigated whether impairment in either of these properties might account for the angiogenic defects observed in the retinas of $T l x^{-/-}$mice.

The overall levels of VEGF mRNA were maintained in $T l x^{-/-}$astrocytes (Figure 6A). Possibly due to this sustained VEGF expression, the hyaloid vessels failed to regress and over time became enlarged and gave rise to numerous endothelial sprouts that grew toward the avascular retina (Figure 6B). Indeed, the sprouting of hyaloid vessels was dependent on VEGF, as these neovessels regressed after the intraocular injection of VEGF Trap (Figure 6B). Interestingly, in $T l x^{-/-}$mice, new vessels derived from the hyaloid were frequently observed to target astrocyte-rich areas of the retina (Figure 6C), suggesting that they were attracted by VEGF produced by these cells. However, upon reaching the retina, these ectopic vessels grew in an apparently random fashion, rather than using the preexisting astrocyte network as a template, as is the case in normal retinal vascular development (Figure 6D). Taken together, these findings strongly suggest that the failure to initiate retinal angiogenesis in $T l x^{-1-}$ mice is not due to a lack of VEGF per se but rather molecules essential for the extracellular matrix scaffolds that form the template for endothelial migration and the initial assembly of the primitive retinal vascular network.

Therefore, we next examined the expression of fibronectin, as this matrix molecule is a principle component of the astrocytederived extracellular scaffold. In $T l x^{-/-}$mice, only a very small number of retinal astrocytes expressed fibronectin mRNA (Figure 


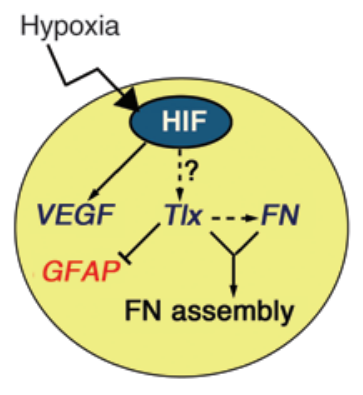

Figure 7

A model for molecular networks regulating the proangiogenic switches in retinal astrocytes. Upon sensing hypoxia, activated HIFs primarily upregulate the transcriptional levels of VEGF and possibly $T / x$. TIx then controls the secondary cascades involved in the extracellular assembly of fibronectin scaffolds, with concomitant repression of GFAP. Once associated with blood vessels, hypoxia-inducible genes supporting the proangiogenic state are downregulated by increasing oxygen concentration. Finally, retinal astrocytes upregulate GFAP and form a bloodretina barrier in cooperation with endothelial cells.

$6 \mathrm{E})$. Consistent with this finding, immunostaining revealed that fibronectin protein was scarce in $T l x^{-/-}$retinas and distributed in a punctate manner (Figure 6F). Intriguingly, in clear contrast to the filamentous and relatively homogenous extracellular deposition of fibronectin around the astrocyte networks in retinas of wildtype and $T l x^{+/-}$mice, fibronectin protein could not be detected in the extracellular spaces around retinal astrocytes in $T l x^{-/-}$mice, but was instead retained in the cytoplasm (Figure 6G). Thus, fibronectin-rich matrices are completely absent from the scaffold formed by $T l x^{-/-}$retinal astrocytes. These observations indicate that $\mathrm{Tlx}$ is essential for the formation of proangiogenic scaffolds through regulation of the extracellular assembly of fibronectin matrices.

\section{Discussion}

Currently it is well established that astrocytes are the major regulators of angiogenesis in the developing retina $(37,38)$. Indeed, retinal astrocytes are found only in species that develop vascularized retinas, such as humans and rodents $(39,40)$. GFAP has been exploited as a representative marker for retinal astrocytes $(41,42)$, but recent studies demonstrated that GFAP is not expressed at appreciable levels in certain astrocyte populations in the developing retina $(22,31,43)$. The GFAP-negative astrocytes have been considered precursors of GFAP-positive astrocytes (43, 44), although the extent to which GFAP expression was a marker for functional heterogeneity remained unclear. In this study, we show that expression of GFAP is inversely correlated with that of 2 major proangiogenic molecules, VEGF and fibronectin. This indicates that GFAP can be exploited as a marker that is negatively correlated with the proangiogenic properties of retinal astrocytes. To identify the molecular mechanisms that regulate the switching of retinal astrocyte phenotype from proangiogenic to angiostatic, we considered the possibility that mechanisms that regulated GFAP expression might also play an integral role in controlling the angiogenic status of retinal astrocytes. Thus, we found that Tlx, a transcriptional repressor of GFAP expression, is an essential component of the molecular network involved in switching the angiogenic phenotype of retinal astrocytes.

Astrocytes are frequently associated with blood vessels in other areas of the central nervous system (25), and the process of neural repair following trauma and the growth of gliomas are characteristically associated with neoangiogenesis. It will therefore be interesting to determine whether GFAP expression is also negatively correlated with the proangiogenic activities of brain astrocytes and whether Tlx is involved in the suppression of GFAP expression in these pathophysiological conditions.

While GFAP is strongly expressed in $T l x^{-/-}$astrocytes, it remains to be determined whether Tlx repression is the sole mechanism that controls GFAP expression in retinal astrocytes. Despite Tlx downregulation after exposure to hyperoxia, GFAP expression was not upregulated beyond the vascularized areas of the retina. This finding suggests that efficient expression of GFAP requires some positive signals in addition to relief of Tlx repression. The precise molecular mechanisms controlling GFAP expression in retinal astrocytes require further elucidation.

Regulation of Tlx expression by oxygen concentration. In addition to retinal astrocytes, Tlx is expressed in neural progenitor or stem cells in the developing forebrain and retina, as well as in adult brain (27, $29,36)$. Although little is known concerning the functional roles of Tlx, recent studies have indicated that Tlx controls cell cycles in these pluripotent, self-renewing cells $(27,29,35,36)$. To date, the mechanisms regulating Tlx expression have also remained unclear. In the present study, we show for what we believe is the first time that $T l x$ expression is transcriptionally controlled by oxygen concentration in retinal astrocytes, thereby restricting its expression in cells in a proangiogenic state. Together with the decreased mitotic activities of $T l x^{-/-}$astrocytes, Tlx expression may partly explain the cell-intrinsic molecular mechanisms underlying the recently reported observation that oxygen regulates mitotic activities in retinal astrocytes (22).

While consensus sequences for hypoxia-responsive elements are found in the $5^{\prime}$ flanking region of the Tlx gene (at $-1277 \mathrm{bp}$ and -2500 bp relative to the transcription start site), we could not detect elevated promoter activities in this region using reporter assays and astrocyte-derived cell lines cultured under hypoxic conditions (mouse cerebellum astrocyte C8-D1A, rat brain astrocyte RCR-1, and human glioma U251, A. Uemura, unpublished observations). Although it remains possible that Tlx transcription is controlled directly by HIFs in native retinal astrocytes, it is also possible that changes in oxygen concentration regulate expression of $T l x$ by an alternate mechanism (5). A related important question is whether oxygen concentration regulates Tlx expression in other types of Tlx-expressing cells. We have observed that retinal neurons continue to express $T l x$ mRNA, even under hyperoxic conditions (A. Uemura, unpublished observations). Therefore, it seems likely that oxygen concentration affects the transcriptional levels of Tlx only in specific cell types and/or under a restricted set of conditions.

Roles of Tlx in the formation of proangiogenic scaffold by retinal astrocytes. In the processes involved in angiogenesis, a number of signaling molecules that modulate endothelial behavior have been identified $(45,46)$, but little is known regarding the molecular mechanisms controlling the other angiogenic processes, especially those underlying the formation of proangiogenic matrix scaffolds. The retinal vascular changes induced by blocking of integrin $\beta 1$ function confirm the importance of endothelial adhesion to matrix scaffolds for the development of the retinal vascular system. Besides providing physical scaffolds for endothelial adhesion and migration, extracellular matrices promote endothelial survival through activating integrin-mediated signal transduction pathways 
(47-50) and establish a concentration gradient of matrix-binding VEGF isoforms $\left(\mathrm{VEGF}_{164}\right.$ and $\mathrm{VEGF}_{188}$ in mice), which guides the direction of sprouting angiogenesis $(19,32)$. It has been reported that hypoxia controls several genes involved in matrix degradation, such as matrix metalloproteinases (5). However, the means by which hypoxia promoted the assembly of proangiogenic fibronectin matrices remained elusive. In the present study, we show that Tlx acts as the major regulator for proper assembly of fibronectin matrices by proangiogenic astrocytes in the developing retina. The overall reduction in the number of fibronectin-expressing astrocytes in $T l x^{-/-}$retinas indicates that Tlx promotes fibronectin transcription. Moreover, the cytoplasmic retention of fibronectin protein by $T l x^{-/-}$astrocytes indicates that Tlx is also critically involved in the extracellular transport of fibronectin. That this fibronectin-rich scaffolding is essential to normal retinal vascular development is strongly supported by observations made in $T l x^{-/-}$mice. Here, normal retinal vascular development fails completely, despite the continued expression of VEGF. In these mice, ectopic vessels sprout from the retained hyaloid vasculature and grow toward the retinal surface. However, upon reaching the retinal surface, these new vessels fail to align themselves along the extant astrocyte network, in contrast to the pattern observed during normal retinal vascular development. Deficiencies in the deposition of fibronectin matrices may also affect the proliferation, adhesion, and migration of astrocytes themselves and partly account for the disorganized architecture of the astrocyte network in $T l x^{-/-}$retina.

It should be emphasized, however, that hypoxia is not the sole factor regulating assembly of fibronectin matrices. In fact, fibronectin is involved in multiple processes from early stages of embryogenesis, and there are many situations where fibronectin assembly should not be affected by the fluctuation of oxygen tension. For example, endothelial expression of fibronectin in retinal vessels may not be maintained by hypoxia. Thus, control of fibronectin deposition by Tlx might be a mechanism for linking hypoxia and scaffold formation specifically in retinal astrocytes. Proangiogenic cells in other tissues that guide endothelial sprouting under hypoxia may have their specific cell-intrinsic mechanisms for fibronectin assembly. Hence, we expect that corresponding molecules with similar functions will be identified in each tissue or organ, which will be potentially beneficial for the advance of technologies to manipulate angiogenesis.

To summarize, we present a model of the molecular network that regulates the angiogenic status of retinal astrocytes during postnatal development (Figure 7). Upon sensing hypoxia, transcription levels of VEGF and Tlx are upregulated, with concomitant upregulation of fibronectin transcription in these astrocytes. Tlx then controls secondary molecular cascades required for the assembly of fibronectin matrices and for regulation of astrocyte behavior through the repression of GFAP expression. Once associated with blood vessels, oxygenation downregulates the hypoxiainducible genes in astrocytes, thereby switching off these proangiogenic activities. Subsequently, astrocytes in their nonangiogenic state continue to collaborate with endothelial cells through other signaling molecules and cell-cell contacts to maintain vascular homeostasis, most notably by promoting the formation and maintenance of the blood-retina barrier.

\section{Methods}

Mice. ICR mice were purchased from SLC Japan, and GFAP-GFP and Tie2-GFP transgenic mice were purchased from the Jackson Laboratory.
Heterozygous Tlx KO mice (26) were crossed to obtain pups that were genotyped by PCR. All animal experiments were approved by the Animal Research Committee of the RIKEN Center for Developmental Biology and were carried out in accordance with the RIKEN guidelines for animal and recombinant DNA experiments.

Histology, immunostaining, and ISH. For whole-mount preparations, retinal cups were isolated and fixed in $4 \%$ paraformaldehyde/PBS overnight. Whole-mount immunohistochemistry procedures were performed as previously described (51). For whole-mount ISH, retinas were briefly digested with proteinase $\mathrm{K}$ and hybridized with digoxigeninlabeled RNA probes. After hybridization, samples were incubated with alkaline phosphatase-conjugated antidigoxigenin antibodies (Roche Diagnostics Corp.), and signals were visualized with nitroblue tetrazolium/5-bromo-4-chloro-3-indoyl phosphate (Roche Diagnostics Corp.). In combined ISH and immunohistochemistry, the antibody labeling was performed after the ISH protocol was completed. Templates for probes (VEGF, 825-1172 of NM_009505: fibronectin, 2466-2831 of BC_025521: Tlx, 683-1840 of NM_152229) were obtained by reverse transcription of total RNA from 4-day old ICR mouse retinas and PCR amplifications. Template for GFAP RNA probe was a gift from James Cohen (King's College London, London, United Kingdom). For cryosections, eyes were frozen in OCT compound (Sakura Finetek and sectioned at a thickness of $10 \mu \mathrm{m}$. Nuclear staining was performed using TO-PRO-3 iodide (Invitrogen Corp.).

$A b s$. Primary Abs for immunohistochemistry were monoclonal antiGFAP (clone G-A-5, Cy3-conjugated; Sigma-Aldrich), PECAM-1 (clone Mec13.3; BD Biosciences - Pharmingen), integrin $\alpha 5$ (clone 5H10-27; BD Biosciences - Pharmingen), integrin $\beta 1$ (clone 9EG7; BD Biosciences - Pharmingen), polyclonal anti-PDGFR $\alpha$ (R\&D Systems), fibronectin (DakoCytomation), type IV collagen (Cosmo Bio Co.), and GFP (Invitrogen Corp.). Secondary Abs were Alexa 488 fluorescence-conjugated goat or donkey IgGs (Invitrogen Corp.) and Cy3- or Cy5-conjugated donkey IgGs (Jackson ImmunoResearch Laboratories). Biotinylated isolectin B4 (Bandeiraea simplicifolia) and Cy3-conjugated antibiotin monoclonal antibody were purchased from Sigma-Aldrich.

Microscopy. Pictures for multilabeling immunohistochemistry were taken with Zeiss laser scanning microscope and LSM510 confocal image software (version 3.2; Zeiss). Pictures for ISH were photographed with Axioplan2 microscope (Zeiss) equipped with a CCD camera (AxioCam HRc; Zeiss) and associated software (AxioVision, version 3.1; Zeiss). In combined ISH immunohistochemistry, photos of the same fields were separately taken in black and white, then signals were colored and merged with Adobe Photoshop 6.0 software.

Intraocular injections. One $\mathrm{mg} / \mathrm{ml}$ of monoclonal hamster anti-mouse integrin $\beta 1$ antibody (clone HM $\beta 1$-1; BD Biosciences - Pharmingen) or VEGF TrapR1R2 (Regeneron Pharmaceuticals) in sterile buffer was injected into the vitreous humor using glass capillary pipettes with a micromanipulator (Drummond Scientific Co.). Control injections of nonspecific hamster IgG or human IgG Fc fragment (Jackson ImmunoResearch Laboratories) were given into contralateral eyes.

Cell sorting and single-step RT-PCR analysis. Retinal cups from 7-day-old Tie2-GFP transgenic mice were digested into single cells with papain and labeled with biotinylated anti-PDGFR $\alpha$ (APA5) (16) antibody followed by allophycocyanin-conjugated streptavidin (Invitrogen Corp.). After cell sorting by BD FACSAria (BD Biosciences), total RNA was isolated from 1000 sorted cells using RNeasy Micro Kit (QIAGEN), and singlestep RT-PCR was performed with OneStep RT-PCR Kit (QIAGEN), using specific primers (Tie2, forward, 5'-TCTTGTGTCTGATGCCGAAAC3'; Tie2, reverse, 5'-GCAGGTAGGAAGGACGCTTGT-3'; PDGFR $\alpha$, forward, 5'-AATCCTGCAGACGAGAGCAC-3'; PDGFR $\alpha$, reverse, 
5'-GCCACCAAGGGAAAAGATTT-3'; fibronectin, forward, 5'-CTCCAGGCGTGGAATACACTTACACCATCC-3'; fibronectin, reverse, 5'GATCAGCATGGACCACTTCTTCCAGAGAGG-3'; $\beta$-actin, forward, $5^{\prime}$-TCGTGCGTGACATCAAAGAG-3'; and $\beta$-actin, reverse, $5^{\prime}$-TGGACAGTGAGGCCAAGATG-3').

Detection of cell proliferation. For BrdU incorporation assays, an intraperitoneal injection of $\mathrm{BrdU}(100 \mu \mathrm{g} / \mathrm{g}$ body weight $)$ was given to P5 neonates 2 hours before enucleation, and the whole-mount retinas were stained by goat anti-PDGFR $\alpha$ polyclonal antibody (R\&D Systems), briefly fixed with $4 \%$ paraformaldehyde, treated with $2 \mathrm{~N} \mathrm{HCl}$ containing $0.1 \%$ Triton X-100 for 60 minutes at $37^{\circ} \mathrm{C}$, and further stained with biotinylated monoclonal antibody for BrdU (clone PRB-1; Chemicon International). BrdU-positive retinal astrocytes at edges of the spreading networks were counted in a fixed area photographed by the $\times 40$ objective. These measurements were performed in 4 random areas and then averaged per retina. For each treatment group, 4 retinas from 4 pups were analyzed. The data were expressed as means \pm SD and were statistically analyzed by 2-tailed Student's $t$ test.

\section{Acknowledgments}

We thank Jim Cohen and Marcus Fruttiger for generously providing GFAP probes and Keiko Funa, Holger Gerhardt, and Masanori Hirashima for critical comments on the manuscript. We are grateful to the Laboratory for Animal Resources and Genetic Engineering in the RIKEN Center for Developmental Biology for the housing of mice. This work was supported by Grants-in-Aid for Scientific Research on Priority Areas (12219209) and the Leading Project grant for Realization of Regenerative Medicine from the Ministry of Education, Culture, Sports, Science and Technology of Japan.

Received for publication June 14, 2005, and accepted in revised form November 11, 2005.

Address correspondence to: Akiyoshi Uemura, Laboratory for Stem Cell Biology, RIKEN Center for Developmental Biology, 2-2-3 Minatojima Minamimachi, Chuo-ku, Kobe 650-0047, Japan. Phone: 8178-306-1924; Fax: 81-78-306-1895; E-mail: auemura@cdb.riken.jp.
1. Hanahan, D., and Folkman, J. 1996. Patterns and emerging mechanisms of the angiogenic switch during tumorigenesis. Cell. 86:353-364.

2. Carmeliet, P., and Jain, R.K. 2000. Angiogenesis in cancer and other diseases. Nature. 407:249-257.

3. Bergers, G., and Benjamin, L.E. 2003. Tumorigenesis and the angiogenic switch. Nat. Rev. Cancer. 3:401-410

4. Cleaver, O., and Melton, D.A. 2003. Endothelial signaling during development. Nat. Med. 9:661-668.

5. Pugh, C.W., and Ratcliffe, P.J. 2003. Regulation of angiogenesis by hypoxia: role of the HIF system. Nat. Med. 9:677-684.

6. Acker, T., and Plate, K.H. 2003. Role of hypoxia in tumor angiogenesis-molecular and cellular angiogenic crosstalk. Cell Tissue Res. 314:145-155.

7. Risau, W. 1997. Mechanisms of angiogenesis. Nature. 386:671-674.

8. Carmeliet, P. 2003. Angiogenesis in health and disease. Nat. Med. 9:653-660.

9. Ferrara, N., Gerber, H.P., and LeCouter, J. 2003. The biology of VEGF and its receptors. Nat. Med. 9:669-676.

10. Stromblad, S., and Cheresh, D.A. 1996. Cell adhesion and angiogenesis. Trends. Cell Biol. 6:462-468.

11. Hynes, R.O. 2002. A reevaluation of integrins as regulators of angiogenesis. Nat. Med. 8:918-921.

12. George, E.L., et al. 1993. Defects in mesoderm, neural tube and vascular development in mouse embryos lacking fibronectin. Development. 119:1079-1091.

13. George, E.L., Baldwin, H.S., and Hynes, R.O. 1997. Fibronectins are essential for heart and blood vessel morphogenesis but are dispensable for initial specification of precursor cells. Blood. 90:3073-3081.

14. Yang, J.T., Rayburn, H., and Hynes, R.O. 1993. Embryonic mesodermal defects in alpha 5 integrin-deficient mice. Development. 119:1093-1105.

15. Bloch, W., et al. 1997. Beta 1 integrin is essential for teratoma growth and angiogenesis. J. Cell Biol. 139:265-278.

16. Fruttiger, M., et al. 1996. PDGF mediates a neuronastrocyte interaction in the developing retina. Neuron. 17:1117-1131.

17. Zhang, Y., and Stone, J. 1997. Role of astrocytes in the control of developing retinal vessels. Invest. Ophthalmol. Vis. Sci. 38:1653-1666.

18. Fruttiger, M., Calver, A.R., and Richardson, W.D 2000. Platelet-derived growth factor is constitutively secreted from neuronal cell bodies but not from axons. Curr. Biol. 10:1283-1286.

19. Gerhardt, H., et al. 2003. VEGF guides angiogenic sprouting utilizing endothelial tip cell filopodia. J. Cell Biol. 161:1163-1177.
20. Stone, J., et al. 1995. Development of retinal vasculature is mediated by hypoxia-induced vascular endothelial growth factor (VEGF) expression by neuroglia. J. Neurosci. 15:4738-4747.

21. Jiang, B., Liou, G.I., Behzadian, M.A., and Caldwell, R.B. 1994. Astrocytes modulate retinal vasculogenesis: effects on fibronectin expression. J. Cell Sci. 107:2499-2508.

22. West, H., Richardson, W.D., and Fruttiger, M. 2005. Stabilization of the retinal vascular network by reciprocal feedback between blood vessels and astrocytes. Development. 132:1855-1862.

23. Janzer, R.C., and Raff, M.C. 1987. Astrocytes induce blood-brain barrier properties in endothelial cells. Nature. 325:253-257.

24. Gardner, T.W., et al. 1997. Astrocytes increase barrier properties and $\mathrm{ZO}-1$ expression in retinal vascular endothelial cells. Invest. Ophthalmol. Vis. Sci. 38:2423-2427.

25. Abbott, N.J. 2002. Astrocyte-endothelial interactions and blood-brain barrier permeability. J. Anat. 200:629-638.

26. Yu, R.T., et al. 2000. The orphan nuclear receptor Tlx regulates Pax2 and is essential for vision. Proc. Natl. Acad. Sci. U. S. A. 97:2621-2625.

27. Shi, Y., et al. 2004. Expression and function of orphan nuclear receptor TLX in adult neural stem cells. Nature. 427:78-83.

28. Young, K.A., et al. 2002. Fierce: a new mouse deletion of Nr2e1; violent behaviour and ocular abnormalities are background-dependent. Behav. Brain Res. 132:145-158.

29. Miyawaki, T., et al. 2004. Tlx, an orphan nuclear receptor, regulates cell numbers and astrocyte development in the developing retina. J. Neurosci. 24:8124-8134.

30. Noto, K., Kato, K., Okumura, K., and Yagita, H. 1995. Identification and functional characterization of mouse CD29 with a mAb. Int. Immunol. 7:835-842.

31. Fruttiger, M. 2002. Development of the mouse retinal vasculature: angiogenesis versus vasculogenesis. Invest. Ophthalmol. Vis. Sci. 43:522-527.

32. Ruhrberg, C., et al. 2002. Spatially restricted patterning cues provided by heparin-binding VEGF-A control blood vessel branching morphogenesis. Genes Dev. 16:2684-2698.

33. Brenner, M., and Messing, A. 1996. GFAP transgenic mice. Methods. 10:351-364.

34. Holash, J., et al. 2002. VEGF-Trap: a VEGF blocker with potent antitumor effects. Proc. Natl. Acad. Sci. U. S. A. 99:11393-11398.

35. Stenman, J.M., Wang, B., and Campbell, K. 2003.
Tlx controls proliferation and patterning of lateral telencephalic progenitor domains. J. Neurosci. 23:10568-10576.

36. Roy, K., et al. 2004. The Tlx gene regulates the timing of neurogenesis in the cortex. J. Neurosci. 24:8333-8345.

37. Wechsler-Reya, R.J., and Barres, B.A. 1997. Retinal development: communication helps you see the light. Curr. Biol. 7:433-436.

38. Gariano, R.F. 2003. Cellular mechanisms in retinal vascular development. Prog. Retin. Eye Res. 22:295-306.

39. Schnitzer, J. 1987. Retinal astrocytes: their restriction to vascularized parts of the mammalian retina. Neurosci. Lett. 78:29-34.

40. Schnitzer, J. 1988. Astrocytes in the guinea pig, horse, and monkey retina: their occurrence coincides with the presence of blood vessels. Glia. 1:74-89.

41. Watanabe, T., and Raff, M.C. 1988. Retinal astrocytes are immigrants from the optic nerve. Nature. 332:834-837.

42. Ling, T.L., Mitrofanis, J., and Stone, J. 1989. Origin of retinal astrocytes in the rat: evidence of migration from the optic nerve. J. Comp. Neurol. 286:345-352.

43. Chu, Y., Hughes, S., and Chan-Ling, T. 2001. Differentiation and migration of astrocyte precursor cells and astrocytes in human fetal retina: relevance to optic nerve coloboma. FASEB J. 15:2013-2015.

44. Mi, H., and Barres, B.A. 1999. Purification and characterization of astrocyte precursor cells in the developing rat optic nerve. J. Neurosci. 19:1049-1061.

45. Rossant, J., and Howard, L. 2002. Signaling pathways in vascular development. Annu. Rev. Cell Dev. Biol. 18:541-573.

46. Yancopoulos, G.D., et al. 2000. Vascular-specific growth factors and blood vessel formation. Nature. 407:242-248.

47. Giancotti, F.G., and Ruoslahti, E. 1999. Integrin signaling. Science. 285:1028-1032.

48. van der Flier, A., and Sonnenberg, A. 2001. Function and interactions of integrins. Cell Tissue Res. 305:285-298.

49. Meredith, J.E., Jr., Fazeli, B., and Schwartz, M.A. 1993. The extracellular matrix as a cell survival factor. Mol. Biol. Cell. 4:953-961.

50. Hynes, R.O. 2002. Integrins: bidirectional, allosteric signaling machines. Cell. 110:673-687.

51. Uemura, A., et al. 2002. Recombinant angiopoietin-1 restores higher-order architecture of growing blood vessels in mice in the absence of mural cells. J. Clin. Invest. 110:1619-1628. doi:10.1172/ JCI200215621. 